\title{
Comparison of Transparent Objects Metrology through Diamond Cutting Edge Radii Measurements
}

\author{
Mansur Akbari ${ }^{\mathrm{a}}$, Wolfgang Knapp ${ }^{\mathrm{a}}$, Konrad Wegener ${ }^{\mathrm{a}, \mathrm{b}}$ \\ a Institute of Machine Tools and Manufacturing, ETH Zurich, \\ Leonhardstrasse 21, 8092 Zurich, Switzerland \\ akbari@alumni.ethz.ch, http://www.iwf.mavt.ethz.ch \\ b inspire AG, ETH Zurich, Technoparkstrasse 1, 8005 Zurich, Switzerland
}

Key words: Metrology; Transparent Objects; Diamond Measurement; Cutting Edge Radius; Comparison of Metrology; Measurement Uncertainty

\begin{abstract}
.
The efficiency of cutting is largely influenced by cutting edge radii. When considering the correct cutting edge radius in the simulations, correct cutting characteristics can be predicted and thus the cutting process optimized. Sharpening the cutting edge of the tool to a specific optimized radius subsequently will improve the service life of the tool, workpiece quality, and performance of cutting. Measuring cutting edge radii of tools already is challenging, especially when measuring cutting edge radii of diamonds cutting tools because of their transparency and their dimension in the micrometer range. Defining a sound framework for selecting the appropriate metrology of transparent objects and clarifying the correct measurement parameters, settings, and proper sample preparation are the main achievement of current work. Tactile profilometer, confocal and focus variation light microscopy, imprinting, scanning electron microscopy (SEM) stereoscopy, and atomic force microscopy (AFM) are used to measure the cutting edge radius of diamonds. The identification of the cutting edge radii are done based on the methodology of the least square circle fit over cutting edge radius, and is determined iteratively. Estimations of uncertainties of the measurements are explained in detail and compared. The same measurement techniques and methodologies can also be applied to measure other transparent or difficult to measure materials. The results of different measurement technologies for the same diamond specimens are compared. In the end, one choice from the utilized measurement methods is suggested based on Analytic Hierarchy Process (AHP), which is one of the methods in Multiple Criteria Decision Analysis (MCDA). The selection process and the proposed set of evaluation criteria can also be applied to other measurements.
\end{abstract}

\section{Introduction}

Optimization of cutting processes without information about cutting edge radii especially in micro-cutting applications is impossible, because cutting edge radii strongly influence the cutting process, i.e. cutting forces, specific cutting energy, cutting temperature, residual stress field in the workpiece, quality of the workpiece after machining and service life of the tool. Klocke [1] has shown that high discrepancies in comparison between simulation and experimental values of feed force and torque in the gun drilling process is due to the nonrealistic assumption of cutting edge radius in the simulation. As shown in $[2,3]$, in turning process feed forces are reduced for sharp cutting edges. Furthermore, in the case of increasing the cutting edge radius in milling, [4] declared that specific cutting energy reduces. Additionally with increasing the cutting edge radius in orthogonal cutting of AISI 316L, it has been proven by [5] that the workpiece temperature increases because of ploughing force on the cutting edge; it is also interpreted as larger edge radius creates larger contact area and 
consequently frictional heat increases. Moreover, it is revealed in [5] that with increasing the cutting edge radius in orthogonal cutting of AISI 316L, the stagnation zone, where the workpiece material starts to split into forming the chip and the new surface of the workpiece, increases. Therefore, the surface of the workpiece is severely plastically strained by cutting with non-sharp tools, which leads to higher residual stresses. Built-up edges, as indicated in [6], can also be formed in sticky or work hardening materials in case of cutting with nonsharp tools, negative rake angle cutting geometry, slow cutting speed, low feed rate and low cutting temperature.

The main problems in the determination of cutting edge radii of diamond tools with light microscopy are transparency and the very small size of diamond, maximum grain size of 710$850 \mu \mathrm{m}$, which makes its measurement very difficult. Thus, measurement noise and uncertainty of the measurement in light microscopy may create problems in the measurement process and generate wrong measurement data. Recently, an optical probe is developed by [7] to measure the cutting edge radius of diamonds. White-light interferometer is also used by [8] to measure the cutting edge radii of diamonds. The problem in using a form and roughness tester is positioning, orienting and fixturing the diamond grain as well as the morphological errors coming from the stylus and fixture. A tactile profilometer is used in [9] for measurement of the edge quality of the polycrystalline diamonds. For SEM, the problem is the non-conductivity of the diamond; therefore always sample preparation have to be done and special consideration must be given to have a homogenous and thin layer of metallic coating on very clean diamond samples. Another problem in SEM is defining the correct orientation for diamond grains and sticking the diamonds properly to the SEM-stubs in which the SEM stub can be used in other measurement systems. Some researchers such as [10], have used SEM to measure the cutting edge of single crystal diamond tools after sharpening the cutting edge for ultra-precision turning processes. The main concern about the measurements with AFM is the large macroscopic surface of the sample which makes it difficult to measure large cutting edge radii of diamonds with small scan ranges, i.e. $3 \mu \mathrm{m}$ in [11]. AFM is used to measure the cutting edge radius of diamonds, such as in $[11,12]$. For the imprinting process, presence of the air bubbles in the negative of diamonds and choice of proper imprinting material to minimize sticking to the sample and deformation after solidification, as well as bearing high elasticity are the main concerns. The evaluation of the imprinting method for production of the positive replicas are explained in detail in [13]. Furthermore, it is shown by [14], that the cutting edge radius of diamonds can also be copied by indenting the tool cutting edge into the surface of a mild metal and then measure the copy of the profile in metal by AFM. However, the problem for the imprinting in metals is the spring back. The issue of transparency in this study is solved by checking different coatings and using the impression material. All the diamonds are rigorously oriented and glued in special fixtures under microscope. The fixtures are accurately mounted in the form and roughness tester. For imprinting process in this study, the air bubbles are removed by a simple injection mold, which is designed just for this application. Furthermore, pressure and cyclic movement is applied to bring the air bubbles to the surface, in addition to selecting the proper imprinting material.

With help of criteria, which cover the advantages and disadvantages of each of the measurement alternative, and applying the AHP method, the optimum measurement technique based on requirement of the user(s), can be selected. A recent research on decision making and evaluating the inconsistencies in pairwise comparisons judgments are analyzed in [15]. Furthermore, most important criterion and least important criterion are used in [16] in a multicriteria decision making method. For the comparison of different metrology methods in this 
study, seven different diamonds are prepared and each of them is measured with five different measurement methods. The analysis of the measurements are done based on one methodology which is the least square circle fit over cutting edge radius that is determined iteratively and is explained in detail in $[17,18]$. Then uncertainties for each measurement are calculated and the measurements are compared. Results of diamond \# 2 from different measurement techniques are shown throughout the paper; however, the result summary using different explained techniques are shown in Fig. 7. The same techniques, which are explained in current work for determination of cutting edge radii of diamonds, can also be used for measurement of other hard to measure geometries and evaluate their surface properties. Measurements of cutting edge radii of some diamonds with particular methods can be found i.e. in $[7-10,12,14]$ and are discussed above. However, characterization of grains for grinding and dressing applications are not reported in literature. Furthermore, the comparison of different measurement methods, calculation of the uncertainties, measurement parameters, and conditions to achieve acceptable results are not found in literature. The same metrology methods can be used for analysis of other transparent objects. Finally, the assessment and selection of suitable metrology can be achieved with the proposed criteria.

\section{Methodology of measurement evaluations}

To evaluate the cutting edge radius, 2D profiles from the measurements are used, as theory of cutting is based on cutting edge radii in a plane square to the cutting edge. This is the reason, why cutting edge radius and not form deviation from a given radius is evaluated. To reduce the uncertainty of the evaluations along the cutting edge, averages of several profiles along the cutting edge are extracted and used in the calculations. The procedure, which is used for cutting edge radius evaluations, is explained schematically in Fig. 1.

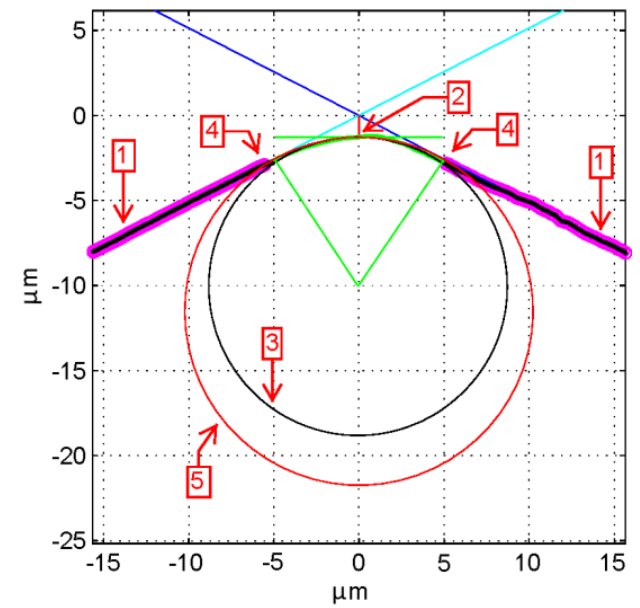

Fig. 1: Methodology of the measurement evaluation based on [17, 18]. The five steps which are explained in Section 2 are shown. For diamond edge \# 2 measured with Leica confocal light microscope; the black circle has a radius of $8.8 \mu \mathrm{m}$ and the finally fitted red circle has a radius of $10.2 \mu \mathrm{m}$.

In brief, this method is based on fitting a least square circle with cutting edge radius that is determined iteratively. It contains five main steps. First, a straight line by least square method is fitted each to the rake and flank faces in the two dimensional profile. These two lines create the wedge angle. Secondly, from the intersection of the bisector of this wedge angle with the profile of cutting edge, one point is defined. Thirdly, a circle is drawn passing through this point and tangential to the two straight lines corresponding to the rake and flank faces. A new upper limit for the least squares straight line fitting is defined from the points where the circle touches the fitted lines. Fourthly, the previous steps are then repeated several times to fulfill 
the criteria of being tangent to the new least square lines and passes the point (similar to the point in step 2) from the intersection of the bisector with the cutting edge profile. Then the two points in the edge profile, where the curvature starts, are defined. Finally, a least square circle is fitted to the profile using all points within the micro geometry limit for the circle. The circle does not necessarily need to touch the fitting lines and its center does not necessarily need to be on the wedge angle bisector. The radius of the fitted circle represents the radius of the rounded cutting edge. More detail about this method can be found in $[17,18]$.

\section{Measurements}

\subsection{General overview in determination of diamond cutting edge radius and sample preparation}

Calibration of a measurement device to assure the results of the measurement is essential. Nevertheless, even after calibration some uncertainties remain due to the nature of the measurement. Therefore special sample preparation or fixtures shall be used to confirm that the measurements can be compared with other calibrated and well-prepared measurement systems. In the current study, seven diamonds with sharp edges, diamond edge \# 1-7 as plotted in Fig. 7, are measured. The selections of the diamonds are based on probability population sampling. In addition, for comparison of different metrology methods, the same cutting edge radii in each of the diamond grains are measured with different measurement devices and software and following concerns are taken into account.

There are some limitations in each measurement system. Since each diamond grain is measured with several measurement systems, a sample preparation, covering all the limitations in different measurement methods is used. The following limitations and steps are considered in the preparation of the diamonds and their fixtures. In Alicona focus variation light microscope, it is better to have the sample in the center of rotation of the rotary unit of the microscope, Real3D ${ }^{\circledR}$. Otherwise, the sample drops out of the measuring window during the rotation process. Moreover, in Form Talysurf ${ }^{\mathrm{TM}}$ Series 2 Taylor-Hobson ${ }^{\circledR}$ tactile profilometer, the stylus with sharp tip moves over the sample and its fixture. If the stylus drops from one of the diamond planes to the fixture after measuring the profile, the diamond tip of the stylus can be damaged. Additionally, if the wedge angle bisector of the cutting edge does not lie approximately on the vertical axis, the form of the stylus creates errors in the measurement profile. By knowing the real shape of the cone, the real shape of the cutting edge can be deduced from the measured data to give the real line of contact points. The same error can also occur if a stylus with large cone angle, such as $90^{\circ}$, is used and the diamond is tilted $30-35^{\circ}$ relative to the plane of stylus-arm at the point of contact with the stylus tip. In SEM, special holders, namely SEM stubs, are used to hold the conductive-coated samples with conductive glue to avoid electrical charge accumulation. Large coating thickness can also create error in the measurement of the cutting edge. To overcome these problems and have a uniform set-up for all the measurements, firstly, holes with diameter and depth, smaller than the diameter of diamonds are drilled exactly on the center of the SEM stubs. The location of drilled hole, in which the diamond is adhered in it, is shown in Fig. 2. The drilled hole in the center of the SEM stub prevents the sample moving out of the field of view during the rotation process with Alicona InfiniteFocus Real $3 \mathrm{D}^{\circledR}$ and in tactile profilometer, reducing the height of the sample, avoids damaging the tip of the stylus while stylus moves over the sample. The target research populations of the diamonds used in this study are truncatedoctahedron single crystalline synthetic diamond (SDB ${ }^{\mathrm{TM}} 1125$ 2025, Element Six e6 ${ }^{\mathrm{TM}}$, ServSix GmbH, Karlstein, Germany). The diamonds have a grain of size D851, i.e. 710-850 
$\mu \mathrm{m}$ that are provided cleaned by the supplier. Secondly, the drilled SEM stubs are ultrasonically cleaned. Subsequently, inert dusting gas HFC-134a (Duster 1671-10S, Techspray, Texas, USA) which is pure and moisture-free is used for removing other microscopic contaminants, which possibly could not be removed by ultrasonic cleaning. This inert gas also removes possible lint, dust, and metallic oxide deposits. Finally, to stick the diamond grains to the SEM pin stub mounts, double coated carbon conductive tabs and Conductive Liquid Silver Paint (G302, 25-50 \% Methyl isobutyl ketone and 50-75 \% Silver, PLANO GmbH, Wetzlar, Germany) are used. It was found that Conductive Liquid Silver Paint is much easier to handle in adjusting the orientation of the diamond edge before solidification of the silver paint comparing to the double-coated carbon conductive tabs. Correct orientation of the diamond grains, helps getting more data points from the planes of the cutting edge in light microscopy. Finally, under microscope the diamonds are glued inside the holes in a way that wedge angle bisector of the cutting edge approximately lies on the vertical axis of the measuring instrument.

The following sections explain the details of each measurement system.

\subsection{Form and roughness tester}

The form and roughness tester is a pointwise measuring system in which a stylus with sharp tip touches the sample with specific force and moves laterally across the topography. Then the displacement of the stylus in vertical direction will be related to the surface topography. As $[19,20]$ have shown, the edge radius of the cutting tools can be measured by this method. In the current study, a Form Talysurf ${ }^{\mathrm{TM}}$ Series 2 (Taylor Hobson $^{\mathrm{TM}}$, Leicester, UK) and diamond stylus with general characteristics of $60^{\circ}$ cone angle and $2^{\mp 0.5} \mu \mathrm{m}$ tip radius is used.

In tactile topography measurements, the observed topography is a convolution of sample topography and tip topography. Therefore, the stylus with $60^{\circ}$ cone angle instead of normal stylus with $90^{\circ}$ cone angle helps to overcome the morphological errors regarding the tip angle of the stylus, which are explained in Section 3.1. The measurement system is connected to a computerized unit with Ultra software (Version 4.6.8) to analyze the surface. In Ultra software, the output of Primary/LS Arc is used to fit a least square arc to the measured data.

Before doing the measurement, the stylus and instrument system are calibrated through measurement of high-precision sphere (radius $79.5361 \mathrm{~mm}$ ) with the Ultra software. Also to be sure that the diamond tip of the stylus did not fracture during the measurement and that the measurement values are correct, the same calibration is repeated after finishing the measurement to check whether the calibration values are within the standard range. For example in current study, the profile depth, $P_{t}$, which is the vertical distance from the lowest to the highest profile point in the primary profile within the evaluation length and is mentioned in [21, 22] must be less than $0.25 \mu \mathrm{m}$. Higher values of $P_{t}$, such as $1 \mu \mathrm{m}$ is an indication of a fractured tip. It is also possible to measure the edge of a razor blade or measure calibrated gauges for topography or surface measurement to ensure that the tip of the stylus is not fractured.

The SEM-stub and its fixture to hold them, as it is explained in Section 3.1, are positioned and monitored while the profilometer moves over the edge of the diamond. The positioning of the fixture is done by caliper and $x-y$ movement of the base. A digital handheld microscope Dino-Lite Premier AM7013MZT (AnMo Electronics Corporation, Taiwan) featuring a five megapixel resolution, is used to monitor the movement of the stylus over the cutting edge to assure reducing the side effects and errors. 


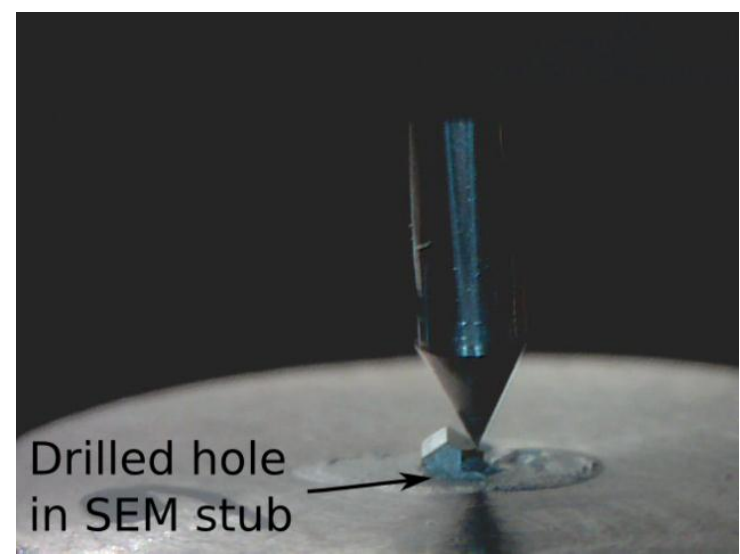

Fig. 2: Snapshot during movement of stylus with diamond tip of $2 \mu \mathrm{m}$ and $60^{\circ}$ cone angle over the cutting edge of the diamond grain. As explained in Section 3.1, this picture also shows the location of the drilled hole in the center of the SEM stub. As it is obvious in the picture, the diamond is properly oriented under microscope before and adhered with silver glue inside the drilled hole in the center of the stub.

\subsection{Light microscopy}

In light microscopy, by increasing the dimension and number of lenses, the magnification of the microscope can increase. Nevertheless, high magnification without very low aberration and diffraction does not guarantee that objects of vanishingly small diameter like diamond edges can be visualized and analyzed properly. As mentioned in [23], there are some ways to improve the aberration and diffraction effects, like proper geometrical shape of lenses, spacing of the lenses and using large aperture lens. Thus, good enough lenses, which are explained in Sections 3.3.1 and 3.3.2, are used to tackle these problems in confocal light microscopy and focus variation light microscopy.

\subsubsection{Confocal light microscopy}

Leica DCM 3D ${ }^{\mathrm{TM}}$ profilometer light microscope (Wetzlar, Germany) is used as another alternative to measure the edge radii. The first step in achieving accurate 3D surface topography from light microscope is adjusting the light source parameters. It was found that setting up light source intensity and focus plane using slit confocal operation mode provided better illumination to capture more data points during confocal microscope operation mode for measuring 3D geometry of the cutting edges. Conventional optical light microscopes can suffer from low contrast images as result of light coming from outside of the focal plane. Confocal microscopy solves this problem by rejecting the scattered light beams coming from above or below the focal plane. Normally the field of illumination and view are limited by pinholes which are positioned on the microscope axis [24]. Slit confocal images utilize a divided aperture and illuminated slit image is scanned across the focal plane. Thus the light scattered or reflected by other planes is blocked by jaws of the slit [25].

Two objective lenses of HCX PL APO (Leica Germany) are used. The first one with 150xmagnification, numerical aperture of 0.95 , field of view of $84.83 \times 63.60 \mu \mathrm{m}, \mathrm{X} / \mathrm{Y}$ optical resolution (lateral diffraction limit) of $0.14 \mu \mathrm{m}$, vertical resolution of less than $2 \mathrm{~nm}$. The second one with 50x-magnification, numerical aperture of 0.9 , field of view of $254.64 \times 190.90$ $\mu \mathrm{m}$, optical resolution of $0.16 \mu \mathrm{m}$, vertical resolution of less than $3 \mathrm{~nm}$. The result of one of these measurements is demonstrated in Fig. 3. 

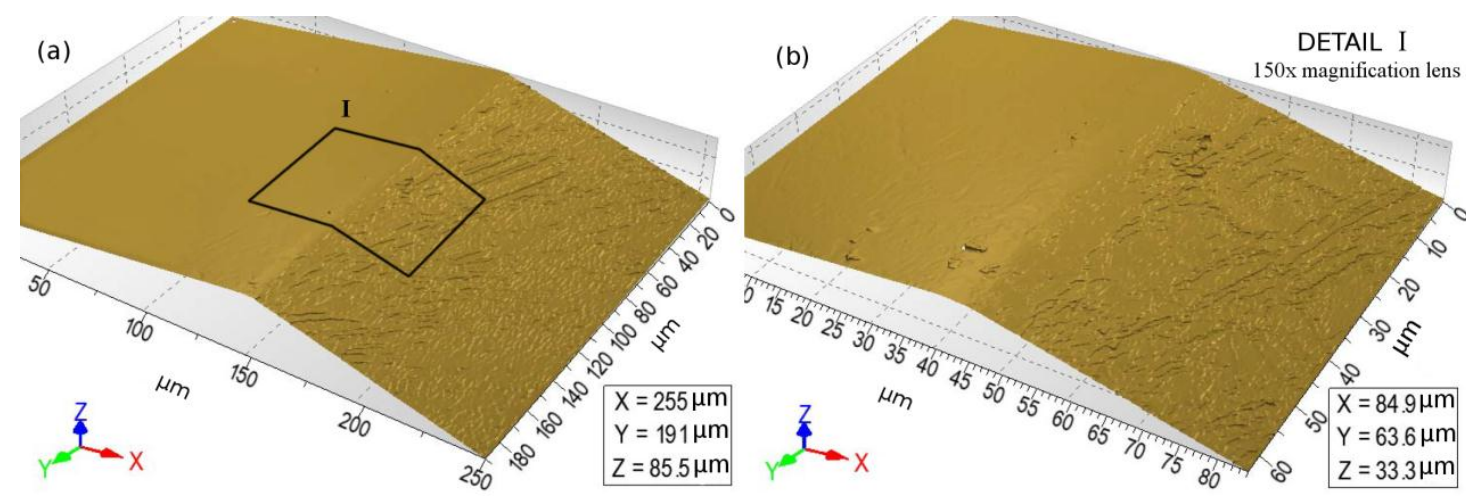

Fig. 3: The topography of the same edges are measured with two different lenses. The volume of the measurement winodows are shown. (a) is captured with 50x magnification lens. The measured and calculated radius is $9.4 \mu \mathrm{m}$. (b) shows the topography of the same edge with $150 \mathrm{x}$ magnification lens with measured and calculated radius of $8.8 \mu \mathrm{m}$. The images are post processed with Leica Map DCM 3D ${ }^{\circledR}$ (Version 6.2.6190).

The results of the measurements with these two lenses for each diamond grain are compared and in case of having the results in the same range for calculations, the results for 50x magnification are used. This is because for 150x magnification, only a smaller window is captured which does not contain enough data points for fitting the least square line as described in Section 2. In best case with this experimental setup, local slopes with more than $70^{\circ}$ inclination angle can be detected. The angles between hexagon and square planes of the synthesized diamond are around $126^{\circ}$. With the help of preparation, positioning, and orientation of the diamonds, which are explained in Section 3.1, none of the square and hexagon planes is perpendicular to the light beam of the microscope and the planes of the diamond had approximately the same angle with respect to the light source. It was found that this setup helps to detect more data points from diamond planes and thus detect the 3D geometry of the edges of the diamond.

\subsubsection{Focus variation light microscopy}

In focus variation light microscopy, Alicona-InfiniteFocus ${ }^{\mathrm{TM}} \mathrm{G} 4$ (Alicona Imaging, Graz, Austria) and the IF-Real3D ${ }^{\mathrm{TM}}$ rotation unit as a fixture are used. Focus variation combines the small depth of focus of an optical system with vertical scanning to provide topographical information from the variation of focus [26]. The light which is focused on specimen via objective lens, scatters strongly in all directions for a surface with diffuse reflective properties or reflects mainly in one direction in case of specular reflection. Since the depth of focus is small, all the rays emerging from the specimen and hitting the objective lens make sharp image only from small regions of the specimens. Thus, the optics moves vertically along the optical axis and continuously captures data from the specimen while each region of the specimen is sharply focused at one of the vertical positions of the scanner. Then by analyzing the variation of the focus along the vertical axis and applying algorithms, the acquired sensored data are converted into 3D information and a true color image with full depth of field can be captured. As mentioned in EN ISO 25178 [26], there are several algorithms to analyze the variation of focus which are usually based on calculation of image sharpness at each scan position. Understanding the algorithms helps capturing better results, which is explained briefly in the following. Typically, an object point is focused more sharply, when neighboring points have a larger variation of image intensity. Each pixel of image has its intensity and the variation of image intensity can be derived from image intensity histogram. Image intensity histogram shows the number of image pixels that display each of the possible discrete intensity values. On the other hand, focus information quantifies the degree of focus 
at defined lateral and vertical position in the surface image. After that, the focus information is saved in a one-dimensional function namely focus information curve for each vertical scan position. Then the height information of the surface is calculated from peak position of the focus information curve. The peak position can be calculated by fitting polynomial or more complex functions to the focus information data and then calculating the maximum or simply using the scan position with maximum focus information.

In current experimental setup, Nikon ${ }^{\mathrm{TM}}$ TU Plan ELWD, objective lens with 20x magnification, numerical aperture of 0.4 and working distance of $19 \mathrm{~mm}$ is used. It was found that due to the transparency of the diamonds, not enough data points can be measured. Gold and carbon coatings of different thicknesses up to approximately $25 \mathrm{~nm}$ are used for the measurements. Also in another set up, the diamonds, which are gold and carbon coated, are painted by Ecoline ${ }^{\circledR}$ (Royal Talens, Apeldoorn, Holland), series 700 in black which is a liquid water color. Immediately after each painting, the high-pressure air nozzle is used to remove thick layers of paints and secure having a homogenous layer thickness. The advantage of this method is its nondestructive procedure and the paint can be easily washed with water. To be sure about the influence of painting, other more viscous liquid water ink in black from Pelikan ${ }^{\mathrm{TM}}$, drawing ink A, grade 17, is used. Then the samples are mounted in IF-Real3D rotation unit to accurately rotate and find the optimum position and orientation for the measurements. Sampling distance is around $440 \mathrm{~nm}$ horizontally and vertically, which created around 1600 points. For the case of using the Pelikan ${ }^{\mathrm{TM}}$ ink for the painting, polarized coaxial light source and external ring light source with maximum light intensity and with the settings in Table 1 are used for the measurements. Adjusting the contrast and gain values is critical to get an image, which is representative of the object. Contrast is the ratio of the difference in intensity between an object and its background relative to the overall background intensity. Gain is used to adjust the number of grey levels included in the resulting image.

Table 1: The important parameters that are used in focus variation light microscope of Alicona for determination of diamond cutting edge in which the gain value shows the detector sensitivity. The criteria to select these parameters are detecting more data points and less noise.

\begin{tabular}{|l|l|l|l|l|l|}
\hline $\begin{array}{l}\text { vertical } \\
\text { resolution }\end{array}$ & $\begin{array}{l}\text { lateral } \\
\text { resolution }\end{array}$ & $\begin{array}{l}\text { Exposure } \\
\text { Time }\end{array}$ & Contrast & Gain & Light source \\
\hline $0.12 \mu \mathrm{m}$ & $1.5 \mu \mathrm{m}$ & $200 \mathrm{~ms}$ & 0.47 & 1 & 1 \\
\hline
\end{tabular}

With all the previously mentioned settings, and checking different parameters it was not possible to avoid having holes on the measured surfaces and cutting edges. Because the layers of coating are not thick enough, in some cases low quality data points resulted, being detrimental for the evaluations. In addition, it is possible that the coating does not cover completely all the diamond surfaces. Moreover, painting a thin layer with homogenous thickness is not successful with the above methods. In current study, higher thickness of coating is not used, because the same diamonds are intended to be measure with several techniques, which would influence the results of other measurement techniques. Thus to overcome this problem, an imprinting process is used which is explained in next section.

\subsubsection{Imprinting method and focus variation light microscopy}

Replicas of objects can be made by imprinting. This facilitates the measuring of hard to measure, hard to access and complex objects, i.e. transparent objects, very narrow deep holes or reducing the mounting and un-mounting the sample. The negative replicas of the object can be made by impression of materials which are categorized by [27] as, plaster, compound, waxes and Zinc oxide/eugenol paste impressions. Based on their application, they have different viscosity, elasticity, solidification time, solidification temperature, expansion 
coefficient, shrinkage and fracture behavior. Adhesion, chemical interaction, and physical locking are other key parameters in fracture of the replica during the demolding. The impression material, which is used here, is Vinyl Polysiloxane, ISO 4823 (type 3, lightbodied) from (Panasil ${ }^{\circledR}$ Contact Plus x-light, Kettenbach GmbH, Eschenburg, Germany). It has 2 minutes working time at $23{ }^{\circ} \mathrm{C}$, total setting time of 4 minutes, $\sim 99.7 \%$ elastic strain recovery and linear dimensional change of $\sim-0.2 \%$ during curing. Since during the imprinting process bubbles of air can be trapped in the surface and influence the surface roughness, a mold with cavities and gates based on [28] is designed to solve this issue based on applying pressure and designing vents to allow escape of trapped air.

The imprinting process is done after finishing all the other measurements with SEM, Taylor-Hobson profilometer and light microscopy to assure that the cleanliness of the diamond samples are not influenced for those measurements. Then the topography of the diamond impression is derived by Alicona focus variation light microscope from negative imprint of the diamond. The imprinting process is done several times to assure having repeatable results.

Table 2: Important parameters that are used in focus variation light microscope of Alicona for determination of imprinted diamond cutting edge. The criteria to select these parameters are detecting more data points and less noise.

\begin{tabular}{|l|l|l|l|l|l|}
\hline $\begin{array}{l}\text { Vertical } \\
\text { resolution }\end{array}$ & $\begin{array}{l}\text { Lateral } \\
\text { resolution }\end{array}$ & $\begin{array}{l}\text { Exposure } \\
\text { time }\end{array}$ & Contrast & Gain & Light source \\
\hline $0.04 \mu \mathrm{m}$ & $1 \mu \mathrm{m}$ & $3.5^{ \pm 0.4} \mathrm{~ms}$ & $1.2^{ \pm 0.6}$ & 2.42 & 1 \\
\hline
\end{tabular}
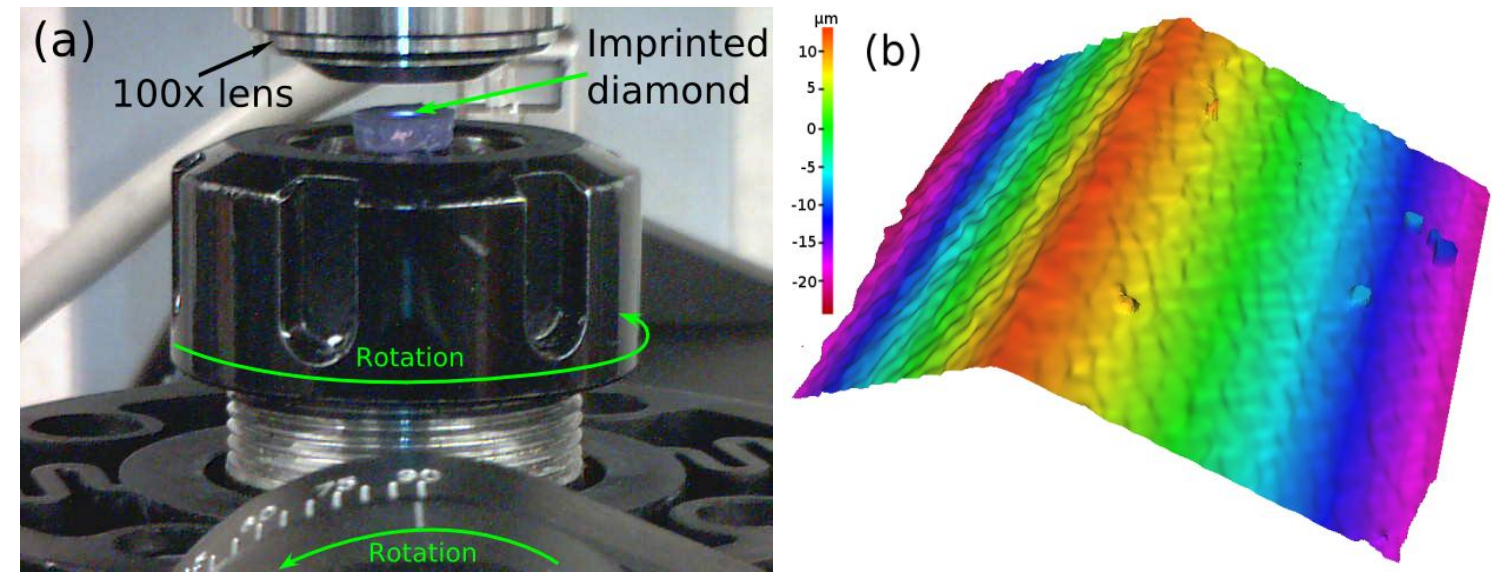

Fig. 4: (a) Measurement of imprinted diamond, which is mounted on 3D rotation unit of Alicona focus variation light microscope; To adjust the wedge angle bisector of the cutting edge to lie approximately on the vertical axis (beam light direction) the rotation axes are used. (b) Inverted, three dimensional topography of imprinted diamond edge \# 2 with 100x magnification lens and with the same procedure as shown in Fig (4.a). The measured and calculated cutting edge radius is $12.7 \mu \mathrm{m}$.

In focus variation light microscope of Alicona, several settings are checked to find the optimum parameters. The working distance of Nikon ${ }^{\circledR}$, LU Plan, ELWD lens with 100xmagnifications and numerical aperture of 0.8 is $3.5 \mathrm{~mm}$. This working distance eases the process of measurement of valleys in the negative imprint of the diamond. It was found that for imprinting material and this specific case study more data points can be captured without using the polarizer of light. Also adaptive control of exposure time/illumination of every single measurement point namely XSmartFlash ${ }^{\circledR}$ is helpful in detecting more data points. The parameters in Table 2 are used in different measurements of the imprints, based on orientation of imprinted planes of diamond with respect to light source and different topographies. Also 
important in the measurement is orienting the model such that two planes of the imprinted edge of the diamond have approximately the same angle with respect to the light source. The measurement set up with focus variation light microscope of Alicona is shown in Fig. 4a. In addition, one of the topographies, which is related to diamond edge \# 2 with radius of 12.2 $\mu \mathrm{m}$ captured by this experimental set up is shown in Fig. $4 \mathrm{~b}$.

\subsection{SEM-Stereoscopy}

SEM can make images with high magnification and depth of field. After the air is pumped out of the SEM chamber, accelerated electrons, which carry significant amount of energy, are emitted from the electron gun. When the accelerated focused incident electrons decelerate in the solid sample, this energy dissipates in the form of secondary electrons (SE), backscattered electrons (BSE), diffracted backscattered electrons, photons, visible light, and heat. Normally SE and BSE are used for imaging. BSE are primary electrons that are ejected from the solid body by an elastic collision of an incident electron and scatter through an angle larger than $90^{\circ}$. Emitted lower-energy electrons resulting from inelastic scattering are called SE. SE can be formed by collisions with the nucleus, where substantial energy loss occurs, or by the ejection of loosely bound electrons from the sample atoms. Since in SEM, electrons travel very close to the optic axis, images with very good spatial resolution and depth of focus can be made.

As mentioned in [23], the SE image displays topographical contrast of the specimen rather than any underlying structure's 3D topographical data points. Therefore, SEM is not enough for evaluation of the topography and radius of cutting edge. With help of Alicona MeX ${ }^{\circledR}$ software, which is based on SEM-Stereoscopy, digital elevation model (DEM) of the topography of the cutting edge is generated by three-dimensional reconstructions. The detail of DEM can be found in [29].

With utilizing the parallax-shift equation from [30] and image processing to detect the features and from disparity of the features in different images, information from depth of image can be derived. To create the DEM in $\mathrm{MeX}^{\circledR}$ software, the feature TriCreator is used. TriCreator utilizes two stereo-pairs SEM images in which one of the images is used twice (three images). Prior to capturing the SEM images, one point in the cutting edge of the diamond should be in the eucentric point of the SEM instrument, i.e. single chosen point in the specimen should be in the same position after tilting the specimen. Thus by tilting and then moving the specimen-stub, one point in the cutting edge brought to the eucentric point. Three images are captured by eucentric tilting of the specimen at $-15^{\circ}, 0^{\circ}$, and $15^{\circ}$. Since the standard eucentric tilting angle is $-10^{\circ}, 0^{\circ}$ and $10^{\circ}$, it is checked to have same measured and calculated edge radii for two different tilting angles of $10^{\circ}$ and $15^{\circ}$. By image matching and entering the calibration parameters in the Alicona $\mathrm{MeX}^{\circledR}$ software, i.e. working distance, pixel size and tilt angle, DEM of the cutting edge radius is derived. In Fig. 5, the SEM images and SEM-stereoscopy, which are made by Alicona $\mathrm{MeX}^{\circledR}$, are demonstrated.

Prior to capturing the SEM images, Argon gas is flooded in the chamber of coating system to avoid influence of air, having homogenous thickness of coating and increasing the efficiency of coating. Then approximately $10 \mathrm{~nm}$ gold is coated on diamonds by Balzers Sputter Coater SCD 050 in $40 \mathrm{~mA}, 410 \mathrm{~V}, 0.05$ mbar vacuum chamber and for sputter time of $70 \mathrm{~s}$. Another setup of the coating with carbon is also applied on uncoated diamonds with

Baltec BAE 120, in $3.5 \times 10^{-5} \mathrm{mbar}, \sim 1800 \mathrm{~V}, \sim 70 \mathrm{~mA}$, gold sputter rate of $\sim 0.1-0.13 \mathrm{~nm} / \mathrm{s}$, sputter time of $88^{ \pm 12} \mathrm{~s}$ and both high vacuum and emissions are adjusted in a way that the sputter rate becomes stable and in the desired range. Then the prepared samples are imaged by SEM (FEI Quanta ${ }^{\mathrm{TM}} 200$ FEG). The SEM-Stereoscopy is made with Tescan ${ }^{\mathrm{TM}}$, Vega 3 XLH. 

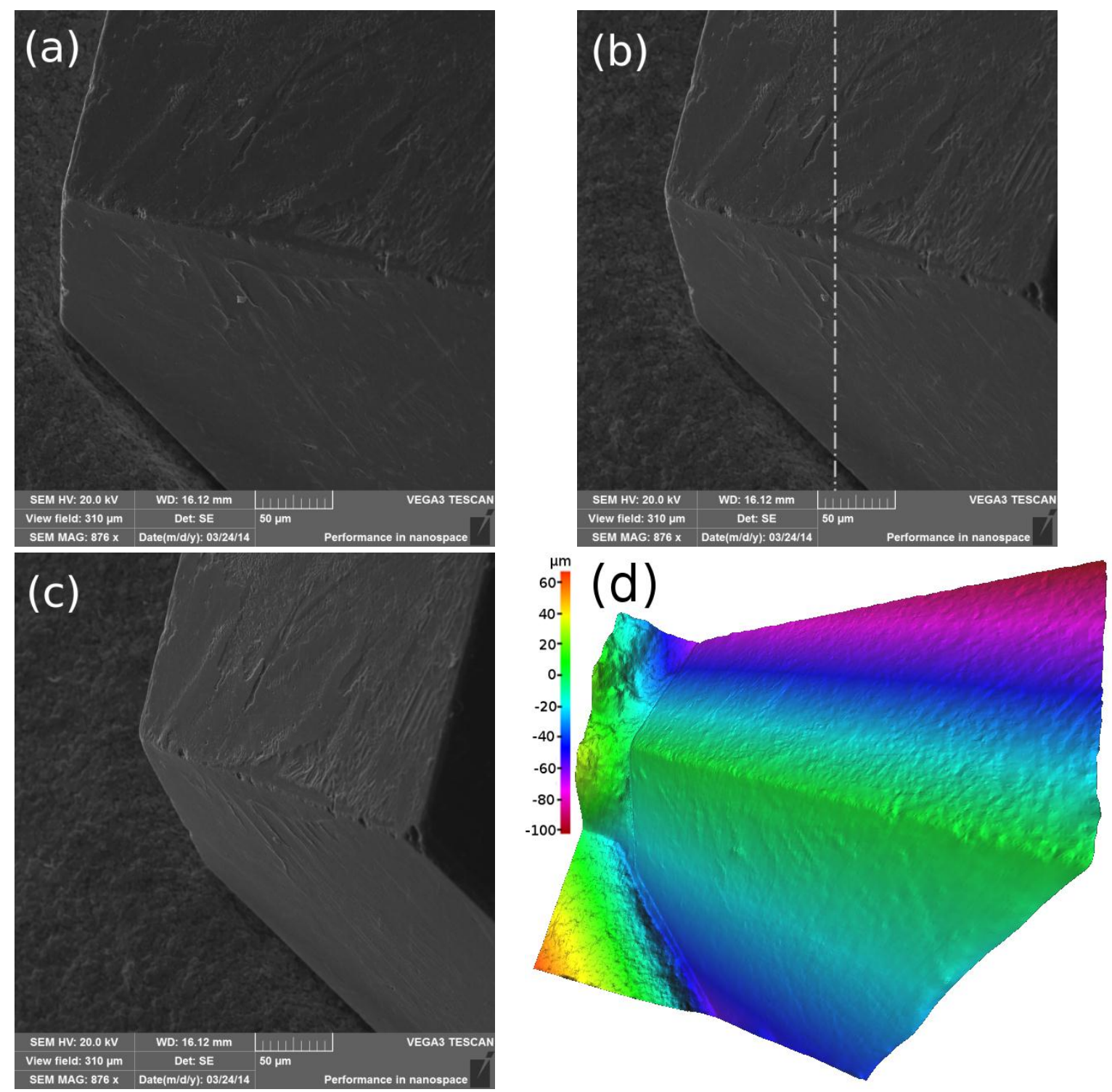

Fig. 5: The procedure of creating three-dimensional topography by SEM-stereoscopy and from eucentric tilting of the specimen and creating three images: a) Tilted $-15^{\circ}$. b) Middle image and zero degree tilted. c) Tilted $+15^{\circ}$. (d) Three-dimensional topography, calculated based on SEM-Stereoscopy by Alicona MeX ${ }^{\circledR}$ software from images in Fig. 5 a,b and c. The axis of rotation is shown in Fig. 5b. The results are related to diamond edge \# 2, and the measured and calculated edge radius is $7.8 \mu \mathrm{m}$.

\subsection{AFM}

With AFM, three-dimensional shape of the surface with details down to nanometer scale can be captured. The measurement of cutting edge radii with an AFM was presented the first time in [11].The advantage of this method is its capability to image all categories of materials without sample preparation and the possibility to operate in air, liquid or vacuum environment. A sharp tip (Berkovic indenter) which is connected to a flexible microcantilever scans the topography, line by line in a contact mode or tapping mode. The bending of cantilever is usually measured by reflecting a laser beam off of the cantilever and onto a split photodiode and the output of which measures the position of the laser spot. The details of AFM can be found in [31]. There are some disadvantages in measuring the radius of diamond edge with contact mode. First, the tip of AFM cantilever may lose its sharpness or might fracture due to the contact with edge of the diamond with high lateral force. The tip and 
diamond might also separate or disengage in the parts of the flank or rake faces that are far from the cutting edge. Loss of contact with the surface can also happen in tapping mode. The Fig. 6 is selected on purpose to also show one of the artifacts which can occur during the measurement even in tapping mode. In the right side of the figure, marked with $\left(^{*}\right)$ sign, one step is captured. That is because the parameters of the AFM such as force are not enough. Therefore, the AFM could not detect the geometry of the root of the topography in right side of the figure. In case of using soft cantilevers to reduce the value of lateral forces and increasing its flexibility, the problem of snapping in of the cantilever arises. Secondly, there is possibility of collision of the body of cantilever with the cutting edge while moving from flank to rake face or vice versa. Thirdly, in contact mode, the friction/ adhesion between the tip and surface of the diamond creates artifacts in the results. Finally, large movement of the tip up and down and accordingly extreme bending of the cantilever may exceed from the range of split photodiode. Hence tapping mode AFM is used for determination of threedimensional structures of the cutting edges of diamonds and afterward calculation of their radii.

The AFM measurements are performed on MFP-3D ${ }^{\mathrm{TM}}$ (Asylum Research, Santa Barbara, CA, USA). The straight cantilevers, which are used in current study, are PointProbePlus ${ }^{\circledR}$ Silicon-SPM-Sensor tips, type PPP-NCHR-W (Nanosensors ${ }^{\mathrm{TM}}$, NanoWorld AG, Neuchatel, Switzerland). The cantilever has $30 \mathrm{~nm}$ thick aluminum coating on its detector side to enhance the reflection of the laser beam; the other specifications of the cantilever and its tip are listed in Table 3.

Table 3: Specification of the cantilever which is used in the AFM measurement [32].

\begin{tabular}{|c|c|c|c|}
\hline Tip radius & Tip height & Stiffness & resonance frequency \\
\hline $10 \mathrm{~nm}$ & $10-15 \mu \mathrm{m}$ & $10-130 \mathrm{~N} / \mathrm{m}$ & $204-497 \mathrm{kHz}$ \\
\hline Thickness & Length & Width & Resistivity \\
\hline $4^{ \pm 1} \mu \mathrm{m}$ & $125^{ \pm 10} \mu \mathrm{m}$ & $30^{ \pm 7.5} \mu \mathrm{m}$ & $0.01-0.02 \Omega \mathrm{cm}$ \\
\hline
\end{tabular}

Since diamonds are mounted on SEM-stubs, a fixture is used to hold the samples on XY scanner plate of AFM instrument. With this fixture, the $\mathrm{Z}$ height of the specimens becomes larger than the standard AFM specimens are. Therefore, three extension legs are used to bring the head of the AFM higher than its normal position and create space for the SEM-stubs and the fixture. After adding the extension legs, with help of spirit level and adjusting screws, the head of AFM is adjusted in horizontal level. By adjusting the focus of lens of light microscope and $\mathrm{X}_{-}, \mathrm{y}$ - camera translation with top view optics, the diamond edge and $\mathrm{X}-, \mathrm{Y}-$ moving of the scanner stage, the cantilever and diamond edge are approximately aligned. Furthermore, the laser is aligned on the cantilever and the deflection of cantilever is adjusted to zero with help of photodetector. Subsequently, by applying the parameters in Table 4, after auto tuning the drive frequency, engaging and bringing down the head of AFM to have an approximate voltage of $80-90 \mathrm{~V}$ across the $\mathrm{Z}$ Piezo crystal, the topographies like the one in Fig. 6 can be measured.

In AFM, the measuring window is small and there is possibility of capturing the geometries like the one marked with $(* *)$ sign in Fig. 6 . Since for the evaluation of the cutting edge radius several profiles along the cutting edge are extracted, such geometries like the one marked with $(* *)$ sign in Fig. 6 will increase the uncertainty of the calculations. Thus, in this case, the measuring window is moved to capture data from other part of the cutting edge without such geometries. 


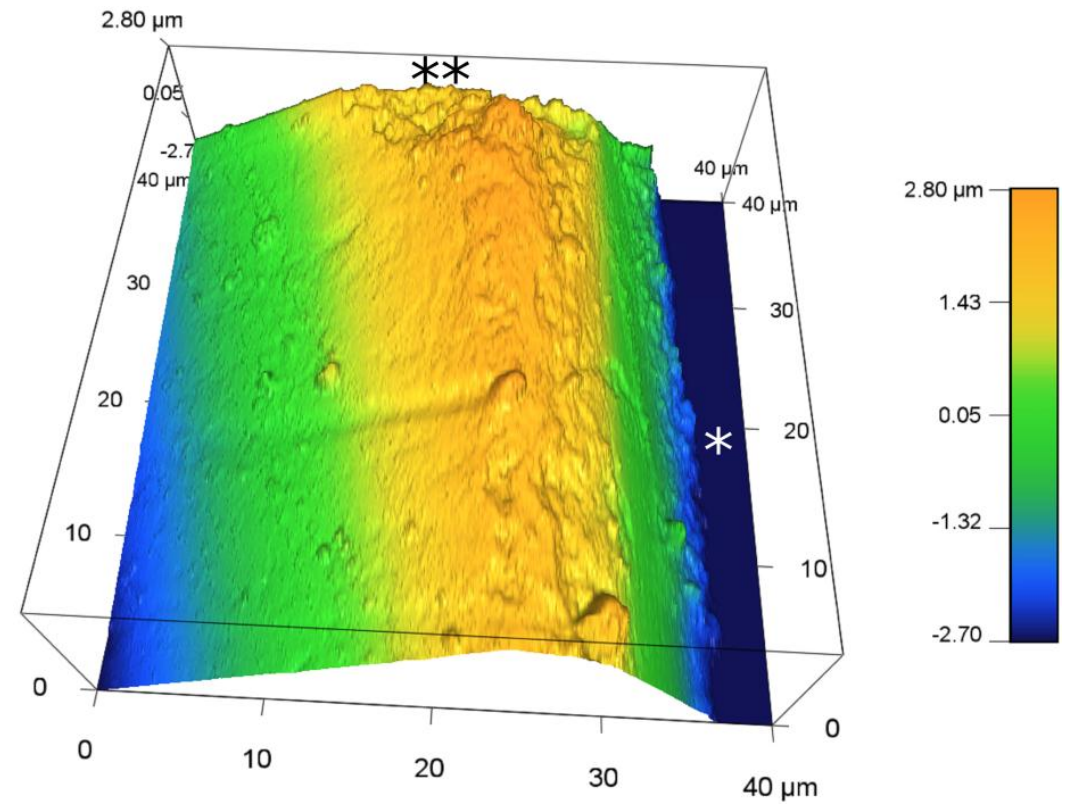

Fig. 6: AFM picture of the diamond cutting edge, the topography is related to diamond edge \# 2 with measured and calculated radius of $10.2 \mu \mathrm{m}$. The geometry which is marked with (**) in the top of the figure is obvious in pictures of SEM and Leica confocal light microscope Fig. 3. This figure is plotted with IGOR Pro-WaveMetrics Inc.

Table 4: The important AFM parameters, which are used for determination of cutting edge topography. The parameters are selected in order to ensure tip contact in each recorded data point and allow the internal control loop of the AFM to follow the topography.

\begin{tabular}{|c|c|c|c|c|}
\hline Scan size & Scan rate & Scan speed & Scan points & Scan lines \\
\hline $40 \times 40 \mu \mathrm{m}$ & $0.1 \mathrm{~Hz}$ & $10.02 \mu \mathrm{m} / \mathrm{s}$ & 2016 & 128 \\
\hline Set points & Integration gain & Feedback filter & Drive amplitude & Drive frequency \\
\hline $650 \mathrm{mV}$ & 10 & $1 \mathrm{kHz}$ & $41.96 \mathrm{mV}$ & $278.185 \mathrm{kHz}$ \\
\hline Voltage & \multicolumn{4}{l}{} \\
\cline { 1 - 4 } $80-90 \mathrm{~V}$ & \multicolumn{4}{|l}{}
\end{tabular}

\section{Results}

\subsection{Estimation of measurement uncertainty}

Uncertainty is the quantitative indication of the quality of the result [33]. More details about the basic concept of uncertainty can be found in $[33,34]$. For the application of the optical measurements techniques to transparent edges, uncertainty evaluations were not found in literature. The measurement uncertainty for each method can be derived from several sources. In the current study, it is classified into six categories that are summarized in Table 5. First uncertainty stems from the instrument, which is specified by the manufacturer. Typically, the manufacturer of the instrument specifies the instrument uncertainty in particular measurement situation, which is the measurement of calibrated gauges for topography or surface measurement under ideal conditions. Second, there is an influence of the environment on the measurement, such as temperature, coatings, dirt, and vibration of the measurement system. Third, it is influenced by fixtures that are used in the measurements. For example, in form and roughness tester, the orientation of the diamonds, angle of the SEMstub with respect to the plane of the machine and the line of the measurement can influence the results of the measurements. Fourthly, uncertainty arises from the object to be measured, 
such as transparency of the diamonds or in case of imprinting, reflection of light from the negative part that is created from the diamond cutting edge. It was also observed that in different locations of one diamond grain and the same edge the radius differs, which can be due to small notches in the cutting edge, deviations of coating, small dirt or to the real topography and this uncertainty can also be considered as object uncertainty. Object uncertainty of $100 \%$ can be for completely transparent, reflective or not possible to measure parts. The object uncertainty is assumed $3 \%$ for the imprinted parts and $2 \%$ for the coated diamonds. Percentage uncertainties are converted to micrometer by multiplication of the calculated cutting edge radius, for each cutting edge, with the percentage uncertainty values.

To reduce the influence of this uncertainty in the calculations, averages of several profiles are used. Fifthly, the uncertainty stems from the computation of cutting edge radius. Finally, there is an influence of the operator in adjusting the correct parameters or provoking other unwanted faults, during the measurements.

Based on [35] for a rectangular distribution with a range of $2 \mathrm{a}$, the standard uncertainty $u_{i}$, can be calculated from

$$
\begin{aligned}
& u_{i}=\frac{a}{\sqrt{3}} \\
& u_{c}=\sqrt{u_{i n s t}^{2}+u_{f i x}^{2}+u_{o b j}^{2}+u_{e n v}^{2}+u_{c o m p}^{2}+u_{o p e}^{2}} \\
& U(k=2)=2 u_{c}
\end{aligned}
$$

The combined uncertainty $u_{c}$ can be calculated from each of the six items of $u_{i}$ as in (1). The expanded measurement uncertainty $U$ is calculated with coverage factor k. Typically $k=$ 2 and the expanded measurement uncertainty is specified as $U(k=2)$ in which $a$ is halfwidth of the rectangular distribution of possible values of input quantity, $u_{\text {inst }}$ is instrument uncertainty, $u_{f i x}$ is fixture uncertainty, $u_{o b j}$ is uncertainty coming from the object, $u_{e n v}$ is environment uncertainty, $u_{\text {comp }}$ is the computation uncertainty and $u_{\text {ope }}$ is uncertainty coming from the operator.

Operator uncertainties for different measurement method are considered based on several parameters. These parameters are the complexity of the measurements and the expertise needed to find the measurement parameters, do the measurements, and post process the results with their corresponding software and possibility of doing mistakes in those steps by the operator. The summaries of operator uncertainties, which are considered in the calculations, are mentioned in Table 5. Operator uncertainty of $100 \%$ can be considered for very complex measurement and unskilled or incapable operator.

For the computation uncertainty, mean value of several measurements are calculated and considered as computation uncertainty for each measurement method. Also for AFM, since diamond \# 6 and 7 showed artifacts are not considered in the calculation of mean value of computation uncertainty of AFM. In Fig. 7 for each diamond edge, this value is calculated separately and mean of these values are mentioned in Table 5. The computation uncertainty of the cutting edge radius also includes the uncertainty due to form deviation.

The influence of vibration on environment uncertainty is neglected, because the measurement instruments are mounted on vibration isolation tables and it is assumed that the isolation tables damp all the vibrations. Also, cleaned diamonds by Element Six Company are used and for the preparation and during the measurements the specimens are kept clean and 
cleaned as explained in detail in Section 3.1. Regarding the possibility of the variation of the coating thickness along the cutting edge, average of the several profiles are considered in the calculations. Therefore, the influence of dirt and deviation of the coating thickness on the environment uncertainty is neglected. For measuring the influence of temperature change, environmental variation error test (EVE) is carried out. In EVE, the range of output of the measurement device is studied in the case of very small or no measurement movement. In EVE, no change on the results could be measured. With considering the thermal expansion coefficient of diamond from [36] and assuming the temperature change, $\Delta T$, of $2{ }^{\circ} \mathrm{C}$ during the measurement, for a diameter of one $\mu \mathrm{m}$ :

$$
u_{i}=\frac{R_{0} \alpha \Delta T}{2 \sqrt{3}}=5.78 \mathrm{e}-07 \mu \mathrm{m}
$$

which is a very small value. The same calculation can be done for other parts of measurement instrument. Thus, the sums of environment uncertainties are neglectable for this case study.

Form Talysurf ${ }^{\mathrm{TM}}$ Series 2, is a tactile surface profiling, therefore the main instrument uncertainty comes from shape of the stylus. The stylus, which is used in current study, has $60^{\circ}$ cone angle and $2 \mu \mathrm{m}$ tip radius. Therefore measuring cutting edge radii smaller than the tip radius will also lead to artifacts, which can be easily calculated and compensated. Furthermore, the diamonds are mounted in a special orientation in which the wedge angle bisector of the cutting edge lies approximately on the vertical axis. As specified in [22], Form Talysurf $^{\mathrm{TM}}$ Series 2 has also the resolution ( $Z$ direction) of $0.64 \mathrm{~nm}$ at a $0.04 \mathrm{~mm}$ measuring range. Instrument uncertainty of tactile profilometer can be derived from measurements on optical flats with two parallel faces. Several parameters should be evaluated with these instrument uncertainty measurements: First of all the straightness of the track, which is defined by gauge; secondly, straightness of the traverse unit; thirdly, perpendicularity of the stylus to the direction of travel; fourthly, the influence of data point resolution, which are recorded by the instrument; fifthly, the repeatability of the measurements. In case of performing the measurement on other previously calibrated verification tools like step-height gauges, the uncertainty of stylus-tip contribution and previously mentioned contribution have to be considered. For the current study, based on a plot of accuracy of measured nominal radii for different nominal radii on page 6-40 of [22], with taking into account the above mentioned points and also the instrument uncertainty of similar instruments, $0.7 \mu \mathrm{m}$ instrument uncertainty has been assumed based on statement of manufacturer Taylor Hobson. For measuring and calculating the uncertainty due to the fixture, two different set of measurements are done. In each measurement the diamond samples are rotated 10 and 20 degrees in $\mathrm{x}^{-}, \mathrm{y}$ - plane (the base plane used for holding the fixture which is perpendicular to the stylus) and positioned accurately by help of digital handheld microscope of Dino-Lite Premier. In tactile profilometer with stylus of $60^{\circ}$ cone angle, the difference between the samples that are 10 and 20 degree rotated and the same samples in 0 degree are $0.6^{ \pm 0.2} \mu \mathrm{m}$ and $0.9^{ \pm 0.3} \mu \mathrm{m}$ respectively. The 10 degrees rotation error of the fixture, error in fixing the diamonds to the SEM stubs and sticking error can happen, which is considered in the calculation of uncertainty. In addition, the radius measurement is affected by length of arc and local form error. The wear of diamond tip of the stylus during the measurement is neglected, which is due to fact that the measurement length is small and contact stresses do not create significant wear or plastic deformation in this short distance. In addition, a new stylus is used for these measurements. Furthermore, after finishing the measurement, the measurement device with the same stylus is calibrated again to be sure about the changes in stylus shape. 
Instrument uncertainty of Alicona-InfiniteFocus ${ }^{\mathrm{TM}} \mathrm{G} 4$ focus variation light microscopy for measurement of edge radii $5 \mu \mathrm{m} \leq R \leq 20 \mu \mathrm{m}$, based on [37], is $1.5 \mu \mathrm{m}$. For confocal light microscope of Leica-DCM 3D and with 50x and 150x confocal objective measurement, based on [38], noise is less than $4 \mathrm{~nm}$ and $2 \mathrm{~nm}$ respectively. Repeatability in the measurement range of these two objectives is better than $0.3 \%$.

With taking into account that the vertical size of the measurement of the feature (in $\mathrm{Z}$ direction) and not the acquisition in $Z$ direction are $1 \mu \mathrm{m} \leq Z \leq 30 \mu \mathrm{m}$, the uncertainty for Leica instrument according to [38] is

$$
u_{i n s t}<\frac{5.5}{\sqrt{Z}} \%
$$

where $\mathrm{Z}$ needs to be given in $\mu \mathrm{m}$, which means for instance, for diameter of $10 \mu \mathrm{m}$, the instrument uncertainty is at least $0.174 \mu \mathrm{m}$.

In AFM, there are mainly four sources of uncertainty, first from probe, secondly from instrument, thirdly from environment such as vibrations and dust, fourthly from image processing. As mentioned in [39], the AFM instrument uncertainty can be calculated from step height, pitch measurement or from complex influence of all AFM parameters and their associated uncertainties in three dimensional measurements. One of the AFM instrument uncertainties stems from optical lever noise or in other words, minimum detectable deflection signal by the optical lever detection system when the cantilever is off the surface. For MFP$3 \mathrm{D}^{\mathrm{TM}}$, this is below $30 \mathrm{pm}$. The optical lever noise limit of $30 \mathrm{pm}$ is considered as vertical resolution. Other AFM instrument uncertainty is from minimum measurable $\mathrm{Z}$ height, which is the system noise and is measured when the cantilever is engaged on the surface. For MFP$3 \mathrm{D}^{\mathrm{TM}}$, this is below $60 \mathrm{pm}$. Certain AFMs have cross coupling between $\mathrm{x}$ and $\mathrm{y}$ drives due to using piezo tube actuation which leads to uncertainties in $\mathrm{x}$ and $\mathrm{y}$ directions. However, in MFP-3D ${ }^{\mathrm{TM}}$, this uncertainty does not exist due to using linear piezo stack in $\mathrm{x}$ and $\mathrm{y}$ directions. Furthermore, the piezo scanner is controlled by closed loop feedback, which eliminates nonlinearities from hysteresis and aging. The piezo of MFP-3D ${ }^{\mathrm{TM}}$ in $\mathrm{Z}$ direction has also more than $15 \mu \mathrm{m}$ sensored travel. The electronic noise of the AFM, originated from the sensors is less than $600 \mathrm{pm}$ for $\mathrm{XY}$ sensors and less than $300 \mathrm{pm}$ for the $\mathrm{Z}$ sensor for MFP-3D ${ }^{\mathrm{TM}}$ as indicated in [40]. In the current study, the largest noise in $\mathrm{Z}$ direction which is $300 \mathrm{pm}$ is considered for instrument uncertainty of AFM. Misalignment of the wedge angle bisector from the vertical axis of the cantilever causes $0.3^{ \pm 0.2} \mu \mathrm{m}$ difference in the results that is considered in the fixture uncertainty.

For evaluation of instrument uncertainty of SEM-stereoscopy method, as explained in detail in [41], the influence of pixel size, number of pixels, working distance and tilt angle can be considered on 3D rendering of standard gates. As stated in [42], the instrument uncertainty of SEM-stereoscopy with Alicona-MeX, in case of measurement of height step of $24 \mu \mathrm{m}$ in ideal condition is $0.14 \mu \mathrm{m}$. Since in SEM-stereoscopy, three-dimensional topography is derived from eucentric tilting of the specimen, fixture uncertainty is neglected.

Finally based on (1) and the data in Table 5, the uncertainties for each measurement and diamond edges in Fig. 7 are calculated. Examples of the expanded measurement uncertainty results, $U(k=2)$, related to diamond edge \# 2, in Fig. 7 and based on (1) for different measurement methods is shown in Table 5. The results of uncertainty calculations are used as a criterion in the evaluation framework in Section 4.3. The results of the uncertainties also propose technological changes in the measurement methods. 
Table 5: Table of resolution, contributors to measurement uncertainty and uncertainty estimation (values are shown for diamond \#2)

\begin{tabular}{|c|c|c|c|c|c|}
\hline 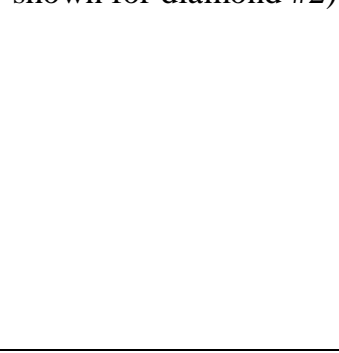 & $\begin{array}{c}\text { AFM } \\
(M F P-3 D)\end{array}$ & $\begin{array}{c}\text { SEM } \\
\text { stereoscopy } \\
(\text { MeX- } \\
\text { Alicona })\end{array}$ & $\begin{array}{l}\text { Tactile } \\
\text { Profilometer } \\
\text { (Taylor } \\
\text { Hobson) }\end{array}$ & $\begin{array}{l}\text { Imprinting } \\
\text { and focus } \\
\text { variation } \\
\text { light } \\
\text { microscopy } \\
\text { (Alicona } \\
\text { 100x) }\end{array}$ & $\begin{array}{c}\text { Confocal } \\
\text { light } \\
\text { microscopy } \\
(\text { Leica } \\
150 x)\end{array}$ \\
\hline $\begin{array}{c}\text { Vertical } \\
\text { Resolution in } \mathbf{n m}\end{array}$ & 0.03 [40] & $3[43]$ & $0.64[22]$ & 10 [44] & $2[38]$ \\
\hline $\begin{array}{c}\text { Instrument } \\
\text { uncertainty in } \mu \mathrm{m}\end{array}$ & 0.0003 & 0.14 & $\approx 0.7$ & $1.5[37]$ & 0.174 \\
\hline $\begin{array}{c}\text { Environmental } \\
\text { uncertainty }\end{array}$ & Assumed 0 & Assumed 0 & Assumed 0 & Assumed 0 & Assumed 0 \\
\hline $\begin{array}{c}\text { Fixture } \\
\text { uncertainty in } \mu \mathrm{m}\end{array}$ & 0.3 & 0 & 0.6 & 0 & 0 \\
\hline $\begin{array}{c}\text { Object } \\
\text { uncertainty in \% }\end{array}$ & 2 & 2 & 2 & 3 & 2 \\
\hline $\begin{array}{c}\text { Computation } \\
\text { uncertainty in } \mu \mathrm{m}\end{array}$ & 0.64 & 1.09 & 1.38 & 1.63 & 1.2 \\
\hline $\begin{array}{c}\text { Operator } \\
\text { uncertainty in \% }\end{array}$ & 0.4 & 0.2 & 0.1 & 0.2 & 0.1 \\
\hline$U(k=2)$ in $\mu \mathrm{m}$ & 1.7 & 2.0 & 3.2 & 4.6 & 2.7 \\
\hline
\end{tabular}

\subsection{Comparison of measurements}

Comparison of different measurement results and methods for the same cutting edge radii of transparent objects was not found in literature. Thus, cutting edge radii of diamonds, which are transparent and hard to measure, are analyzed with several methods. The cutting edges of the diamonds from truncated-octahedron single crystalline synthetic diamond (SDB ${ }^{\mathrm{TM}} 1125$ 2025, Element Six e6 ${ }^{\mathrm{TM}}$, ServSix GmbH, Karlstein, Germany) can be classified as sharp (diamond edge \# 1-7 in Fig. 7) and with chamfer edges, based on probability population sampling. Diamonds with chamfer edges are not in the scope of this study. The measurements of diamond edges \# 1-7 in Fig. 7, show that the cutting edge radii of the diamonds with sharp edges are between 5 and $15 \mu \mathrm{m}$. All the measurement results for each cutting edge, when considering measurement uncertainties, overlap each other. For diamond edge \# 7, the AFM results do not overlap with confocal light microscopy, tactile profilometer, and SEMstereoscopy results. The reason is, for large edge radii like diamond edge \# 7 , the body of cantilever interacts with cutting edge radius and makes AFM method not suitable for determination of large cutting edge radii. Therefore, all the other measurements are acceptable. In addition, the results show that there is not a specific order for the maximum and minimum values or an order for the other measurement methods between extrema. Because of this fluctuation of results, it is hard to rank the applied measurements.

AFM, comparing to SEM-stereoscopy, do not need special treatment, thus do not damage the sample by coating. Furthermore, AFM does not need vacuum; therefore, it is cheaper and can work in different environment. The resolution in AFM is also higher than in SEMstereoscopy. Nevertheless, for measurement of cutting edge radii, AFM is limited to 
maximum image of $150 \times 150 \mu \mathrm{m}$ depending of the AFM instrument whereas in SEM the image window limitation is in order of $\mathrm{mm}$. Moreover, incorrect choice of tip and cantilever in AFM, leads to image artifacts. AFM is not as fast as SEM-stereoscopy and in case of increasing the speed, uncertainties due to temperature influence and change of properties of the cantilever may happen. Also in AFM, cross-talks between coordinates and piezoelectric hysteresis phenomenon may create artifacts. The results in Fig. 7 show that AFM measurement of cutting edge radii is suitable for sharp edges like diamond edge \# 1 and not suitable for blunt edges like diamond edge \# 7. In measurement of cutting edge radius, the AFM tip is much smaller than diamond edges with radius larger than around $10 \mu \mathrm{m}$. Thus for measurement of large radii of cutting edges of the diamonds, instead of interaction of sharp tip of the cantilever with diamond edge, the body of the cantilever interacts with the cutting edge radius of diamond and leads to non-realistic results.

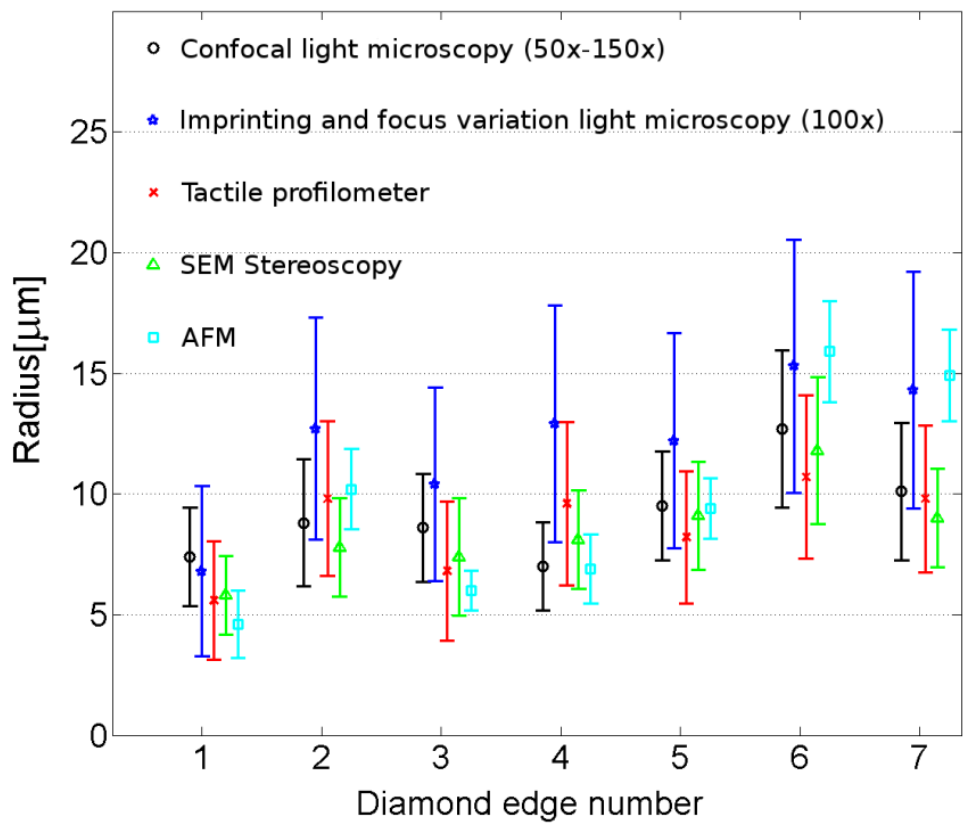

Fig. 7: Comparison of the results of the cutting edge radius measurements: The results show that the measured values of the cutting edge radius varies between about 5-10 $\mu \mathrm{m}$. The uncertainties which are shown in the graphs are calculated based on the theory in Section 4.1.

Uncertainty of measurement with confocal light microscopy and 150x magnification lens is far more less than focus variation light microscopy with 100x magnification lens. Despite the fact the results with confocal light microscope has less uncertainty than focus variation light microscopy, the very small working distance of confocal light microscope makes its measurement harder than focus variation light microscopy. However, after setting up the experiment, the measurements with confocal light microscopy is faster than focus variation light microscopy. The measurement time with tactile profilometer is less than other measurement methods and its results are also in acceptable range, however it needs special considerations that are explained in previous sections. Some of these considerations are setting up the experimental setup, as explained in sections 3.1, calibration, and selection of the proper stylus, as explained in section 3.2, and taking into account the uncertainties, which are explained in section 4.1. For assessment of the measurement methods/instruments, evaluation of the measurement results and selecting a measurement technique, a methodology is needed which is explained in the following section.

\subsection{Selection of the most feasible measurement method based on AHP}


Each of the measurement methods in current work has advantages and disadvantages. With help of Multiple Criteria Decision Analysis (MCDA), based on taking explicitly into account of multiple criteria, such as those mentioned in Table 6, judgment and finally decision on selecting the appropriate measurement method from number of measurement alternatives can be made. Analytic Hierarchy Process (AHP) is a method in MCDA, based on solution of eigenvalue problem, which hierarchically decomposes the complex system and defines a network structure.

The structure of the decision problem is a hierarchy consisting of three levels: the goal of the decision, which is the selection of the most feasible measurement method, at the top level, followed by a second level consisting of the criteria by which the measurement method alternatives, located in the third level, will be evaluated. The relation within the structure is established by simple pairwise comparisons. Normalized principal eigenvectors of the matrices of the pairwise comparisons develop weightings. As mentioned in [45], calculation of the eigenvectors of the matrix of ratio comparisons assures having a consistent result. Then alternatives are evaluated according to the weightings to find the highest ranking. The highest ranking defines the goal of the decision. In current study the AHP method is used, which is implemented in Expert Choice Comparion ${ }^{\mathrm{TM}}$ software (Expert Choice Inc., Arlington VA, USA).

To represent the degree of importance of the pairwise comparisons, fundamental scales are used in comparison of homogeneous elements. The alternatives in the AHP analysis are the five measurement methods that are mentioned in Fig. 7 and the criteria with their weighting are listed in Table 6 . To reduce the complexity of the decision making process and the number of criteria, several criteria are combined. For example, the criterion of "cost of measurement" contains need of special infrastructure for the instrument, purchase cost of the instrument, cost of the extra facilities, total cost of ownership (TCO), utensils, and materials, time of measurement and sample preparation. The criterion "No need of high expertise to do the setup of the experiment and having user-friendly software" is yet another combined criterion. In general, the results of measurement are reliable in case of having high accuracy with low uncertainty. Thus as shown in Table 6, the highest weight factor is considered to be $30 \%$, for accuracy of the measurement method with low uncertainty. However, the overall cost of the measurement has to be reasonable to make the measurement feasible. Therefore, a weight of $25 \%$ is set.

After defining matrix of pairwise comparisons, which has the order of the number of alternatives $\times$ the number of criteria and multiplying to the column vector matrix of weightings of the criteria, the overall priorities for ranking the alternatives is calculated. The elements of the pairwise comparisons matrix that has the order of the number of alternatives $x$ the number of criteria are the ordinate in Fig. 8 for each criterion. The elements of the column vector matrix of weightings of the criteria are in Table 6 . To have a consistent result for the vector of the priorities of the most feasible measurement method, eigenvalue formulation is considered. The eigenvalues of the matrix of the ratio comparisons of the weightings that has the eigenvector matrix of the weightings of the criteria, in Table 6, proves the consistency in the vector of priorities.

With performance sensitivity, which is the derivative of the quality function with respect to the criterion value, the importance and influence of weights of the criteria on ranking of alternatives can be analyzed. Performance sensitivity of this decision is shown in Fig. 8 and can be seen that at the end, recommendations are given for selection of tactile measurement with profilometer as plotted in Fig. 8. This is recommended mainly because of providing good enough resolution and low measurement cost which includes initial cost, infrastructure, TCO, 
time of measurement and preparation time. In addition, tactile measurement of diamond edges is often referred to as introducing wear to the measuring tip, which cannot be confirmed by this study. The same methodology and criterion that is explained in this section can be used by readers to assess and select a measurement method, especially for other transparent objects.

Table 6: Criteria and their corresponding weights, which are used in AHP. To reduce the size of pairwise comparison some criteria are combined to one, such as cost of measurement.

\begin{tabular}{|l|c|}
\hline Criterion & Weight \\
\hline C1: Uncertainty and accuracy & 0.3 \\
\hline C2: Resolution & 0.2 \\
\hline C3: Cost of measurement (Initial, infrastructure, TCO, time, preparation) & 0.25 \\
\hline C4: Bigger sample size can be measured in one step and easily & 0.05 \\
\hline C5: Working distance & 0.01 \\
\hline C6: Working environment & 0.05 \\
\hline $\begin{array}{l}\text { C7: (No need of) High expertise to do the setup of the experiment and having } \\
\text { user- friendly software }\end{array}$ & 0.1 \\
\hline C8: Robustness of the instrument & 0.04 \\
\hline
\end{tabular}

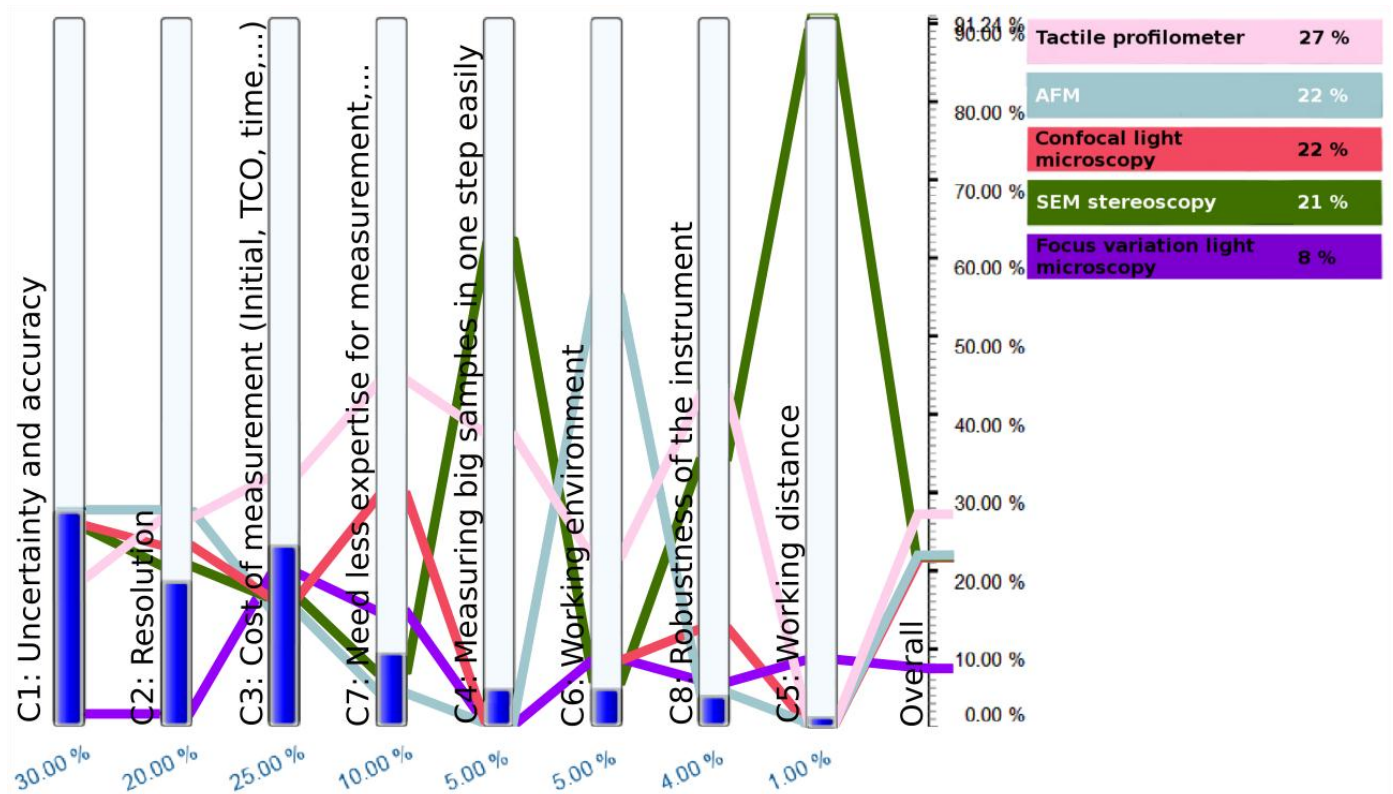

Fig. 8: Performance sensitivity of AHP decision-making process. The plot also shows how each measurement method is rated with respect to each criteria and the overall result. The blue columns indicate the weightings of criteria. Tactile profilometer is the method that is recommended for this task. Moreover, the plot shows the sensitivity of weights of each criterion to the final ranking of alternatives.

\section{Conclusions}

For the application of the measurement of cutting edge radii of transparent materials, no clear guidelines exist on what can be expected from the measurement methods i.e. optical methods. Therefore, comparisons of different measurement methods are performed and a framework for criteria of measurement tool selection is setup and filled with concrete values. Cutting edge radii of diamond grains, which are transparent and in micro scale, are measured with several methods. Confocal and focus variation light microscopy, imprinting process, tactile profilometer, SEM stereoscopy and AFM are used to do these measurements. The cutting edge radii of synthesized truncated-octahedron single crystalline synthetic diamond 
$\left(\mathrm{SDB}^{\mathrm{TM}} 1125\right.$ 2025, Element Six e6 ${ }^{\mathrm{TM}}$, ServSix GmbH, Karlstein, Germany) with sharp edges are between 5 and $15 \mu \mathrm{m}$. The calculation of uncertainties for each method is discussed in detail. The limitations and advantages of each method are indicated and at the end, the measurement parameters, which are used to capture good enough data with uncertainties, are shown so that the readers can use them to do their measurements. Furthermore, the sample preparations and fixtures that are used to do these measurements are explained. The same techniques can be applied to other transparent or hard to measure samples. Since each metrology alternative has advantages and disadvantages, for assessment of the measurement methods and selecting a measurement technique a methodology is needed. In current study, AHP method is used and criteria are suggested for assessment of the measurement methods. Recommendations are given for tactile profilometer based on main criteria of uncertainty, costs, resolution, and simple to use as well as the selection of the weights of criteria.

The study carried out here yields some experience even to enhance the different methods in their accuracy, which are summarized as follows: A micro positioning system to allow for the $3 \mathrm{D}$ rotation of the sample to find the optimum orientation of the sample. Using such a system, the measurements of Taylor-Hobson tactile profilometer and Leica confocal light microscope can be improved. For tactile profilometer, using stylus with smaller cone angle than $60^{\circ}$ with sharper tip radius is suggested, similar to the one that has been used in this study, i.e. $0.2 \mu \mathrm{m}$ radius stylus tip with a $45^{\circ}$ cone angle. In tactile profilometer, lower speed of the traverse unit and detecting more data points helps deriving the topography with more precision especially in the extrema of the profile, like the cutting edge radius. In light microscopy, oil immersion technique or facilitating oil immersion objectives can improve the resolution of the measurement. To overcome the problem of transparency of the diamonds for focus variation light microscopy, it is possible to coat thicker layer of carbon or gold and then subtract the thickness of the coated layer in the calculations of the cutting edge radius. Uncertainty of the measurements can thus also be improved. For example, the computation uncertainty can be improved by improving the algorithm of the calculation or since the average of several profiles are used in the calculations, by having more accurate data points from the measurement.

Acknowledgements The authors would like to thank the Swiss National Science Foundation for the financial support under the number 200021-117847. We also acknowledge Element Six Company (ServSix GmbH) for providing the diamonds. Specially, M. Akbari would like to thank Prof. Ralph Spolenak, group of Micro and Nanosystems-ETHZ, EMEZ (electron microscopy ETH Zurich), André Röthlisberger, Armin Tobler, Dr. Fredy Kuster, Albert Weber and Sandro Wigger.

\section{References}

1. F. Klocke, M. Abouridouane, K. Gerschwiler, and D. Lung, 3D Modelling and Simulation of Gun Drilling, in Modelling of Machining Operations, J. C. Outeiro, Editor. 2011. p. 12-19.

2. C. F. Wyen and K. Wegener, Influence of cutting edge radius on cutting forces in machining titanium. CIRP Annals - Manufacturing Technology, 2010. 59(1): p. 93-96.

3. B. Karpuschewski, K. Schmidt, J. Prilukova, J. Beňo, I. Maňková, and N. T. Hieu, Influence of tool edge preparation on performance of ceramic tool inserts when hard turning. Journal of Materials Processing Technology, 2013. 213(11): p. 1978-1988.

4. M. Arif, M. Rahman, and W. Y. San, A study on the effect of tool-edge radius on critical machining characteristics in ultra-precision milling of tungsten carbide. International Journal of Advanced Manufacturing Technology, 2013. 67(5-8): p. 1257-1265.

5. M. N. A. Nasr, E. G. Ng, and M. A. Elbestawi, Modelling the effects of tool-edge radius on residual stresses when orthogonal cutting AISI 316L. International Journal of Machine Tools \& Manufacture, 2007. 47(2): p. 401-411. 
6. Metalcutting Technical Guide: Turning, Milling, Drilling, Boring, Toolholding; . 2005: Handbook from Sandvik Coromant.

7. S. JANG, Y. SHIMIZU, S. ITO, and W. GAO, Development of an optical probe for evaluation of tool edge geometry. Journal of Advanced Mechanical Design, Systems, and Manufacturing, 2014. 8(4): p. 110.

8. F. Qin, K. Chou, D. Nolen, R. G. Thompson, and W. Ni, Cutting edge radius effects on diamond coated tools. Trans NAMRI/SME, 2009. 37: p. 653-660.

9. T. B. Thoe, D. K. Aspinwall, M. L. H. Wise, and I. A. Oxley, Polycrystalline diamond edge quality and and surface integrity following electrical discharge grinding. Journal of Materials Processing Technology, 1996. 56(1-4): p. 773-785.

10. I. Miyamoto, T. Ezawa, and K. Nishimura, Ion beam machining of single-point diamond tools for nanoprecision turning. Nanotechnology, 1990. 1(1): p. 44.

11. D. A. Lucca, Y. W. Seo, and R. Komanduri, Effect of Tool Edge Geometry on Energy Dissipation in Ultraprecision Machining. CIRP Annals - Manufacturing Technology, 1993. 42(1): p. 83-86.

12. W. J. Zong, Y. H. Huang, Y. L. Zhang, and T. Sun, Conservation law of surface roughness in single point diamond turning. International Journal of Machine Tools and Manufacture, 2014. 84(0): p. 58-63.

13. M. Henerichs, M. Egeter, T. Liebrich, R. Voß, and K. Wegener, Evaluation of the IWF-Wunder Reproduction Method for Generating Positive Replica. International Journal of Automation Technology, 2014. 8: p. 49-56.

14. X. P. Li, M. Rahman, K. Liu, K. S. Neo, and C. C. Chan, Nano-precision measurement of diamond tool edge radius for wafer fabrication. Journal of Materials Processing Technology, 2003. 140: p. 358-362.

15. S. Siraj, L. Mikhailov, and J. A. Keane, Contribution of individual judgments toward inconsistency in pairwise comparisons. European Journal of Operational Research, 2015. 242(2): p. 557-567.

16. J. Rezaei, Best-worst multi-criteria decision-making method. Omega, 2015. 53(0): p. 49-57.

17. C.-F. Wyen, Rounded cutting edges and their influence in machining titanium. Fortschritt-Berichte VDI. Reihe 2: Fertigungstechnik. Vol. 685. 2012: VDI Verlag. 220.

18. C.-F. Wyen, W. Knapp, and K. Wegener, A new method for the characterisation of rounded cutting edges. The International Journal of Advanced Manufacturing Technology, 2012. 59(9-12): p. 899-914.

19. C. Dold, M. Henerichs, L. Bochmann, and K. Wegener, Comparison of ground and laser machined polycrystalline diamond (PCD) tools in cutting carbon fiber reinforced plastics (CFRP) for aircraft structures, in Fifth Cirp Conference on High Performance Cutting 2012, K. Wegener, Editor. 2012. p. 178-183.

20. C. F. Wyen, D. Jaeger, and K. Wegener, Influence of cutting edge radius on surface integrity and burr formation in milling titanium. International Journal of Advanced Manufacturing Technology, 2013. 67(1-4): p. 589-599.

21. DIN, Geometrische Produktspezifikation (GPS) - Oberflächenbeschaffenheit: Tastschnittverfahren Benennungen, Definitionen und Kenngrößen der Oberflächenbeschaffenheit, in DIN EN ISO 4287 (2010-07-00). 2010, Deutsches Institut für Normung, Beuth Verlag GmbH, 10772 Berlin: Deutschland

22. Taylor/Hobson, Form Talysurf Series 2, Operator's Handbook. Taylor/Hobson precision: Leicester, England, LE4 9JQ.

23. R. F. Egerton, Physical principles of electron microscopy. 2007: Springer.

24. S. Inoué, Foundations of Confocal Scanned Imaging in Light Microscopy, in Handbook Of Biological Confocal Microscopy, James B. Pawley, Editor. 2006, Springer US. p. 1-19.

25. C. Koester, A Comparison of Various Optical Sectioning Methods: The Scanning Slit Confocal Microscope, in Handbook of Biological Confocal Microscopy, Jamesb Pawley, Editor. 1990, Springer US. p. 207-214.

26. DIN, Geometrische Produktspezifikation (GPS) -Oberflächenbeschaffenheit: Flächenhaft -Teil 606: Merkmale von berührungslos messenden Geräten(Fokusvariation) (ISO/DIS 25178-606:2013); in DIN EN ISO 25178-606. 2013, Deutsches Institut für Normung.

27. J. F. McCabe, Applied dental materials, ed. Angus W. G. Walls. 2008, Oxford: Oxford : Blackwell Publishing.

28. V. R. Dominick, Injection molding handbook, ed. Dominick V. Rosato. 2000, Boston: Boston : Kluwer Academic Publishers.

29. F. M. David, Digital elevation model technologies and applications : the DEM user manual, 2nd. edition, ed. David F. Maune. 2007, Bethesda: Bethesda : ASPRS.

30. L. J. Whittlesey, Computer Techniques of Acquiring and Producing Three-dimensional Videos. 2007, Stephen F. Austin State University. p. 87. 
31. H. Greg, Atomic force microscopy : understanding basic modes and advanced applications, ed. Greg Haugstad. 2012, Hoboken, NJ: Hoboken, NJ : Wiley.

32. Nanosensors, Catalog of PointProbe Plus, Silicon-SPM-Probes. 2014: Switzerland.

33. JCGM, Evaluation of measurement data - Guide to the expression of uncertainty in measurement. 2008, BIPM (Bureau international des poids et mesures).

34. S. Bell, A Beginner's Guide to Uncertainty of Measurement. Measurement Good Practice Guide No. 11 (Issue 2). 2001, Teddington, Middlesex, United Kingdom, TW11 0LW: The National Physical Laboratory(NPL).

35. ISO/IEC, GUIDE 98-3:2008(E) Uncertainty of measurement, in Part 3: Guide to the expression of uncertainty in measurement (GUM:1995). 2008: Geneva, Switzerland. p. 120.

36. M. Akbari, S. Buhl, C. Leinenbach, R. Spolenak, and K. Wegener, Thermomechanical analysis of residual stresses in brazed diamond metal joints using Raman spectroscopy and finite element simulation. Mechanics of Materials, 2012. 52: p. 69-77.

37. Alicona, Measurement uncertainty of InfiniteFocus G4 and InfiniteFocus SL / IF-EdgeMaster. 2014, Alicona Imaging GmbH: Graz, Austria. p. 2.

38. Leica Microsystems, Technical Instruction. 2014.

39. A. Yacoot and L. Koenders, Recent developments in dimensional nanometrology using AFMs. Measurement Science \& Technology, 2011. 22(12).

40. Asylum Research, AFM in Biology, Class 2010, Asylum Research, Editor. 2010: Santa Barbara, CA.

41. L. Carli, G. Genta, A. Cantatore, G. Barbato, L. D. Chiffre, and R. Levi, Uncertainty evaluation for three-dimensional scanning electron microscope reconstructions based on the stereo-pair technique. Measurement Science and Technology, 2011. 22(3): p. 035103.

42. Alicona, How Accurate is MEX and How to Improve Accuracy. 2008, Alicona Imaging GmbH: Graz, Austria.

43. Elektronen Optik Service GmbH. TESCAN VEGA SEM Specifications. 2014 [cited 2014 June]; http://www.eos-do.com/html/vega01.html].

44. Alicona, Alicona infinite focus, The magazine about Alicona-metrology in IF-20102011-4. 2014, Alicona Imaging $\mathrm{GmbH}$.

45. L. S. Thomas and L. V. Luis, Models, methods, concepts \& applications of the analytic hierarchy process, ed. Thomas L. Saaty and Luis L. Vargas. Vol. 175. 2012: New York : Springer. 